\title{
Photonic crystal (PhC) nanowires for infrared photodetectors
}

\begin{abstract}
We report the Photonic Crystal $(\mathrm{PhC})$ nanowires performance for potential phototodetectors integration application. The refractive index of $\mathrm{PhC}$ can be tailored to guide specific resonance wavelength precisely. This paper presents the numerical approach of $1 \mathrm{D} \mathrm{PhC} \mathrm{with}$ 12 periodic holes to observe the range of stop band acquired, transmission and the quality factor of the resonance wavelengths. By splitting the holes equally with a range of cavities from 440 to $450 \mathrm{~nm}$, the stop band observed are between 1.5 to $2.1 \mathrm{\varepsilon m}$. By varying the cavity length, the value of resonance wavelengths and quality factors observed have also changed. The introduction of $442 \mathrm{~nm}$ cavity shows the highest transmission but the lowest Quality factor (Q-factor) where both are observed at 0.87 and 284 respectively. The values indicate a good confinement of light in the waveguide designed thus enabling wavelength selectivity for photodetectors application in highly sensitive wavelength selection application.
\end{abstract}

Keyword: Cavity; Graphene; Nanowires; Photodetectors; Photonic band-gap; Photonic crystal; Transmission 